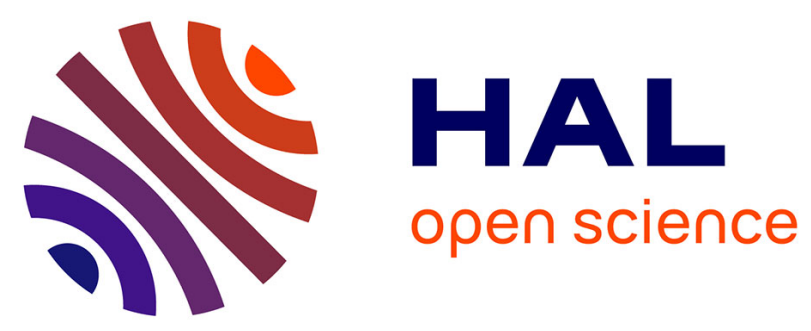

\title{
Observation and deconvolution of a unique EPR signal from two cocrystallized spin triangles
}

Logesh Mathivathanan, Yiannis Sanakis, Raphael G Raptis, Philippe Turek, Athanassios K Boudalis

\section{- To cite this version:}

Logesh Mathivathanan, Yiannis Sanakis, Raphael G Raptis, Philippe Turek, Athanassios K Boudalis. Observation and deconvolution of a unique EPR signal from two cocrystallized spin triangles. Physical Chemistry Chemical Physics, 2021, 23 (26), pp.14415-14421. 10.1039/D1CP01965A . hal-03321393

\section{HAL Id: hal-03321393 https://hal.science/hal-03321393}

Submitted on 17 Aug 2021

HAL is a multi-disciplinary open access archive for the deposit and dissemination of scientific research documents, whether they are published or not. The documents may come from teaching and research institutions in France or abroad, or from public or private research centers.
L'archive ouverte pluridisciplinaire HAL, est destinée au dépôt et à la diffusion de documents scientifiques de niveau recherche, publiés ou non, émanant des établissements d'enseignement et de recherche français ou étrangers, des laboratoires publics ou privés. 


\title{
Observation and deconvolution of a unique EPR signal from two cocrystallized spin triangles
}

Logesh Mathivathanan, ${ }^{1,2}$ Yiannis Sanakis, ${ }^{3}$ Raphael G. Raptis, ${ }^{1}$ Philippe Turek, ${ }^{4}$ Athanassios K. Boudalis* ${ }^{4,5}$

${ }^{1}$ Department of Chemistry and Biochemistry and the Biomolecular Sciences Institute, Florida International University, Miami, FL 33199, USA.

${ }^{2}$ Department of Chemistry, GITAM Institute of Science, Gandhi Institute of Technology and Management (Deemed to be University), Visakhapatnam, AP, India 530045

${ }^{3}$ Institute of Nanoscience and Nanotechnology, NCSR “Demokritos”, 15310 Aghia Paraskevi, Athens, Greece.

${ }^{4}$ Institut de Chimie de Strasbourg (UMR 7177, CNRS-Unistra), Université de Strasbourg, 4 rue Blaise Pascal, CS 90032, F-67081 Strasbourg, France.E-mail: bountalis@unistra.fr.

${ }^{5}$ Institut de Science et d'Ingénierie Suparamolaiculaires - ISIS, 8 allée Gaspard Monge, BP 70028, F67083 Strasbourg Cedex, France

\begin{abstract}
A 16-line pattern has been theoretically predicted, but hitherto not reported, for the Electron Paramagnetic Resonance (EPR) spectrum of antiferromagnetically coupled $\mathrm{Cu}^{\text {II }}$ triangles experiencing isotropic exchange of isosceles magnetic symmetry. Now, the crystallization of such a triangular species and its X-ray structure determination in a polar space group, $R 3$ (No. 146), has enabled its single crystal EPR study. Its detailed magnetic susceptibility, X- and Q-band, powder and single crystal EPR spectroscopic study reveals the effect of molecular structure and of Dzyaloshinskii-Moriya interactions (DMI) on the $g_{\|}, g_{\perp}$ and $A_{\|}$parameters of the spectrum; DMI is considered for the first time in such a context. Moreover, careful analysis of the spectrum allows the deconvolution of two slightly different cocrystallized magnetic species.
\end{abstract}




\section{Introduction}

Spin triangles have recently received renewed attention in various contexts, such as their proposed use as decoherence-free, electrically controlled spin-chirality qubits, ${ }^{1-3}$ a proposal which has received validation by the recent demonstration of magnetoelectric couplings within them. ${ }^{4-7}$ The basis of this application hinges upon the presence of Dzyaloshinskii-Moriya interactions (DMI), which are responsible for the emergence of spin chirality ${ }^{8,9}$ and for the spin currents that are responsible for exchange-induced electric polarization. ${ }^{6}$ Hence, identification and treatment of DMI in the presence of various other interactions is a key prerequisite in developing such an application framework.

The theory that describes the magnetism of spin triangles has been developed over the past seven decades, starting from the first use of the isotropic Heisenberg-Dirac-van Vleck Hamiltonian Hamiltonian for polynuclear complexes by Kambe. ${ }^{10}$ Since then, the theoretical framework has been enhanced with the inclusion of isosceles distortions of the isotropic exchange, ${ }^{11} \mathrm{DMI}^{12}$ hyperfine interactions $^{13,14}$ and dipolar inter- ${ }^{15,16}$ and intramolecular ${ }^{17}$ interactions. These have been recently reviewed by one of us. ${ }^{18}$

The experimental signatures of spin triangles are very sensitive to interdependencies among each of these terms. As an example, the value of the molecular $g$-tensor elements lying in the triangle plane $\left(g_{\perp}\right)$ depend critically on the relation between the symmetry of the isotropic interactions (isosceles deformation) and DMI. ${ }^{19}$ The EPR spectra of spin triangles are typically analysed within the framework of a spin Hamiltonian including at least an isosceles distortion of the isotropic exchange and a Dzyaloshinskii-Moriya term:

$$
H=-2 J\left(\hat{\mathbf{S}}_{1} \hat{\mathbf{S}}_{2}+\hat{\mathbf{S}}_{1} \hat{\mathbf{S}}_{3}\right)-2 J^{\prime} \hat{\mathbf{S}}_{2} \hat{\mathbf{S}}_{3}-2 \mathbf{G}\left(\hat{\mathbf{S}}_{1} \times \hat{\mathbf{S}}_{2}+\hat{\mathbf{S}}_{2} \times \hat{\mathbf{S}}_{3}+\hat{\mathbf{S}}_{3} \times \hat{\mathbf{S}}_{1}\right)+\mu_{B} \mathbf{H} \Sigma \mathbf{g}_{i} \hat{\mathbf{S}}_{i}
$$

where $\mathbf{G}$ is the DMI pseudo-vector. ${ }^{18}$ This Hamiltonian explains the $g$ anisotropy and, assuming $G_{x}=$ $G_{y}=0$, it can be analytically calculated that the effective $g$ values are given by $g_{\|, \text {eff }}=g_{0 \|}(2)$ and $g_{\perp, e f f}=g_{0 \perp} \sqrt{\frac{\delta^{2}-(h v)^{2}}{\Delta^{2}-(h v)^{2}}}$ (3), where $\delta=2\left|J-J^{\prime}\right|, \Delta=\left(\delta^{2}+\left(2 \sqrt{3} G_{z}\right)^{2}\right)^{1 / 2}$ (for $S=1 / 2$ spins), and $g_{0 \| \mid}, g_{0 \perp}$ are the components of the intrinsic $g$-tensors of the individual ions.

Hyperfine interactions, described by the additional term $H_{i}^{\mathrm{HF}}=\hat{\mathbf{I}}_{i} \tilde{\mathbf{A}} \hat{\mathbf{S}}_{i}$, have been neglected in this general model, as their occurrence depends on the magnetic nuclei of each metal, i.e. their nuclear spin, natural abundance etc. Moreover, the magnetic symmetry of Hamiltonian (1) impacts the hyperfine pattern of EPR spectra due to the redistribution of the spin density over the metal sites, ${ }^{14}$ while it also 
impacts the EPR signatures of dipolar interactions as we showed in the case of a H-bonded dimer of spin triangles. ${ }^{16}$

Theoretical analysis of a purely isotropic ( $\mathbf{G}=0$ ) copper(II) triangles predicts that hyperfine couplings to the three ${ }^{63 / 65} \mathrm{Cu}(I=3 / 2)$ should result in 24 resonances that break down to different groupings as a function of magnetic symmetry: in the case $|J|>|J|$ (case I) the $\left|S_{23}, S_{T}\right\rangle$ ground state will be the $|1,1 / 2\rangle$ one, giving rise to a 16-line pattern, while case $|J|<|J|$ (case II) will stabilize the $|0,1 / 2\rangle$ multiplet which will give rise to a 4-line pattern, not unlike that of mononuclear $\mathrm{Cu}^{\text {II }}{ }^{14}$

Reports of 4-line patterns have appeared for spin-coupled antiferromagnetic $\mathrm{Cu}_{3}{ }_{3}$ complexes, ${ }^{20-22}$ albeit without a rigorous analysis of their origin. In previous work we reported a H-bonded dimer of

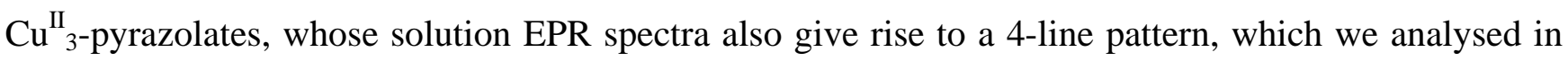
detail. ${ }^{16}$ An 8-line pattern in the powder spectrum of a $\mathrm{Cu}^{\mathrm{II}} 7$ aggregate was observed and was attributed to a spin coupling scheme involving two strong and one weak $J$ couplings, within a fully isotropic model. $^{23}$

A 16-line pattern has not been reported to date, whereas the additional impact of DMI has never been taken into consideration in explaining the EPR experimental signatures of hyperfine interactions. While ${ }^{57} \mathrm{Fe}$ Mössbauer spectroscopy has demonstrated the impact of DMI on hyperfine interactions in the case of ferric triangles, ${ }^{24}$ such a demonstration has not been accomplished to date using EPR spectroscopy.

Herein, we report powder and single-crystal X- and Q-band EPR studies of a complex previously characterized as $(\mathrm{PPN})_{3}\left[\mathrm{Cu}_{3}\left(\mu_{3}-\mathrm{O}\right)\left(\mu-4-\mathrm{Cl}-\mathrm{pz}_{3} \mathrm{Cl}_{3}\right] \mathrm{Cl}\right.$ by ambient temperature $\mathrm{X}$-ray structure determination (4-Cl-pz ${ }^{-}=4$-chloropyrazolate; $\mathrm{PPN}^{+}=$Bis(triphenylphosphine)iminium) ${ }^{25}$

\section{Results and discussion}

A previous single-crystal structural study of complex $\mathbf{1}$ had revealed that it crystallizes in the polar, trigonal $R 3$ space group, with a crystallographic 3-fold rotation axis passing through the center of the triangle of a $C_{3 v}$ point-group symmetry. Preliminary EPR studies of this complex had revealed intriguing powder spectra, with a complex hyperfine pattern (vide infra). Eager to elucidate these rare signals, we undertook single-crystal EPR studies, which also entailed additional X-ray diffraction characterization.

Interestingly, the new low-temperature crystallographic analysis now revealed two electron density peaks on the 3-fold rotation axis in the proximity of $\mu_{3}-\mathrm{O}^{2-}$, which are assigned to $\mu_{3}-\mathrm{Cl}^{-}$and $\mu_{3}-\mathrm{OH}^{-}$ bridges with occupancy factors of 0.22 ; correspondingly, this reduces the occupancy factor of $\mu_{3}-\mathrm{O}^{2-}$ to 
0.78. The crystallographic determination has therefore been reinterpreted as stemming from the cocrystallization of two complex double salts, $\quad(\mathrm{PPN})_{3}\left[\mathrm{Cu}_{3}\left(\mu_{3}-\mathrm{O}\right)(\mu-4-\mathrm{Cl}-\mathrm{pz})_{3} \mathrm{Cl}_{3}\right] \mathrm{Cl} \cdot \mathrm{H}_{2} \mathrm{O}$ $\left((\mathrm{PPN})_{3}[\mathbf{1 a}] \mathrm{Cl} \cdot \mathrm{H}_{2} \mathrm{O}\right) \quad$ and $\quad(\mathrm{PPN})_{3}\left[\mathrm{Cu}_{3}\left(\mu_{3}-\mathrm{OH}\right)\left(\mu_{3}-\mathrm{Cl}\right)(\mu-4-\mathrm{Cl}-\mathrm{pz})_{3} \mathrm{Cl}_{3}\right] \mathrm{Cl} \cdot \mathrm{H}_{2} \mathrm{O} \quad\left((\mathrm{PPN})_{3}[\mathbf{1 b}] \mathrm{Cl} \cdot \mathrm{H}_{2} \mathrm{O}\right)$ resulting in a mixed phase with formula $(\mathrm{PPN})_{3}\left[\mathrm{Cu}_{3}\left(\mu_{3}-\mathrm{O}\right)_{0.78}\left(\mu_{3}-\mathrm{OH}\right)_{0.22}\left(\mu_{3}-\mathrm{Cl}\right)_{0.22}(\mu-4-\mathrm{Cl}-\right.$ $\left.\mathrm{pz})_{3} \mathrm{Cl}_{3}\right] \mathrm{Cl} \cdot \mathrm{H}_{2} \mathrm{O}$ (or $(\mathrm{PPN})_{3}[\mathbf{1 a}]_{0.77}[\mathbf{1 b}]_{0.23} \mathrm{Cl} \cdot \mathrm{H}_{2} \mathrm{O}$, or simply $\mathbf{1}$ ). The complex anions $\mathbf{1 a}$ and $\mathbf{1 b}$ of the two salts are shown in Figure 1. Both molecules share the same symmetry elements and, consequently, are aligned with their magnetic axes parallel to each other (Figure S1).

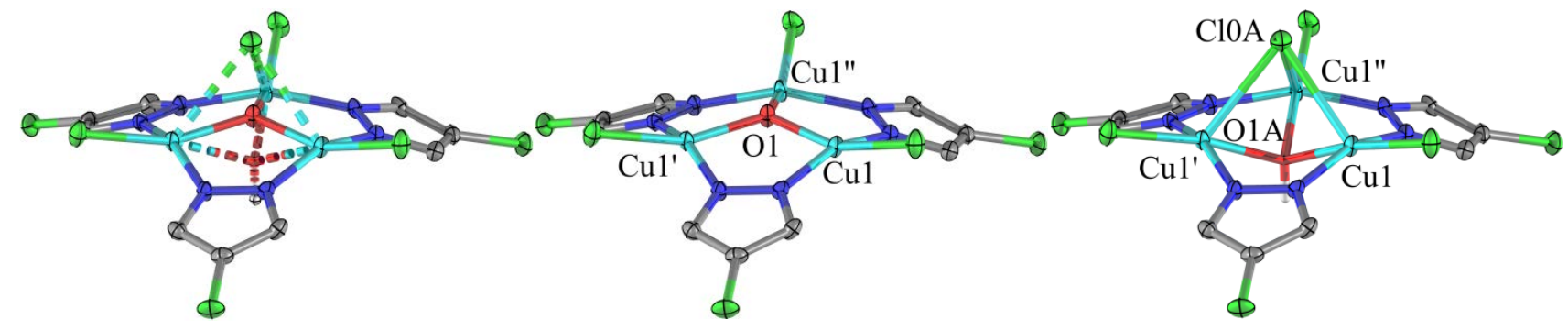

Figure 1. POV-Ray thermal ellipsoid plots (50\% probability level) of the cocrystallized anionic complexes in $(\mathrm{PPN})_{3}[\mathbf{1 a}]_{0.78}[\mathbf{1 b}]_{0.22} \mathrm{Cl} \cdot \mathrm{H}_{2} \mathrm{O}$. Left: A composite representation of the molecules indicating the relative orientations of the different bridging moieties. Center: Dianion 1a. Right: Dianion 1b. Symmetry operator symbols: ' $=-y, x-y, z ; "=-x+y,-x, z$.

Magnetic susceptibility studies of 1 reveal that at $300 \mathrm{~K}$, the $\chi_{M} T$ product is $1.37 \mathrm{~cm}^{3} \mathrm{~mol}^{-1} \mathrm{~K}$, comparable to the theoretically expected value for three non-interacting $S=1 / 2$ ions $\left(1.36 \mathrm{~cm}^{3} \mathrm{~mol}^{-1} \mathrm{~K}\right.$, $g=2.2$ ). Upon cooling this value decreases, reaching a value of $0.19 \mathrm{~cm}^{3} \mathrm{~mol}^{-1} \mathrm{~K}$ at $2 \mathrm{~K}$. Given the fact that two species are present, $\mathbf{1} \mathbf{a}$ and $\mathbf{1 b}$, with comparable, but not identical, expected magnetic behaviours, magnetic susceptibility studies cannot discern their individual contributions, thus indicative fits were carried out considering a single species. An equilateral model without DMI could not account for the low- $T$ behaviour, usually affected by DMI. In contrast, inclusion of DMI afforded a remarkable improvement, even assuming an equilateral magnetic symmetry, with best-fit parameters $J=J^{\prime}=-49.8$ $\mathrm{cm}^{-1}$ and $\left|G_{z}\right|=16.0 \mathrm{~cm}^{-1}$ (Figure 2). 


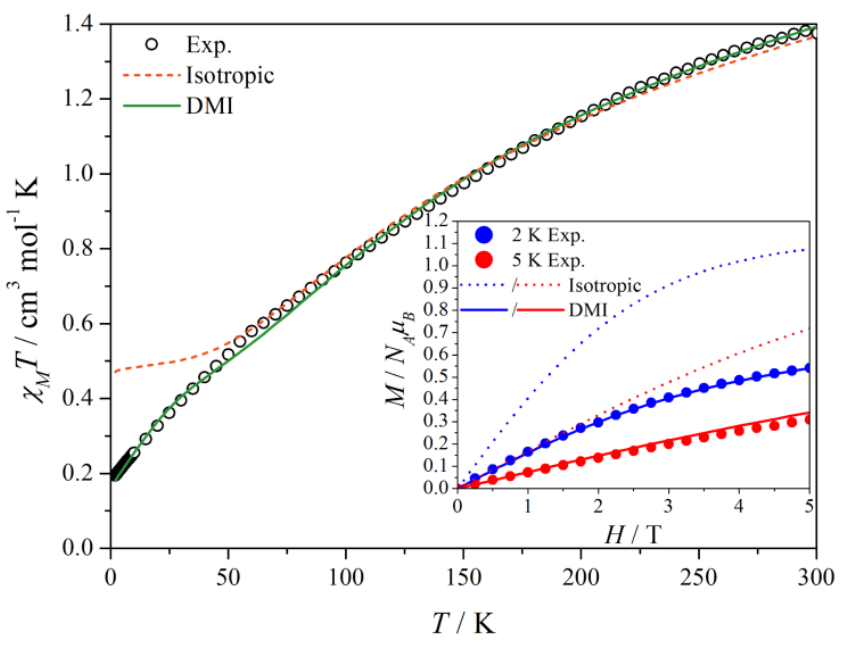

Figure 2. Fits to the magnetic susceptibility ( $5 \mathrm{kG}$ ) and isothermal magnetization (2 and $5 \mathrm{~K}$ ) data assuming a single-phase sample and: (i) an isotropic $(\mathbf{G}=0)$ model (dashed lines), or (ii) an anisotropic $(\mathbf{G} \neq 0)$ model incorporating DMI (solid lines). Best-fit parameters for the anisotropic model: $J=J^{\prime}=$ $49.8 \mathrm{~cm}^{-1},\left|G_{z}\right|=16.0 \mathrm{~cm}^{-1}, g=2.29, \chi_{\mathrm{TIP}}=859 \times 10^{-6} \mathrm{~cm}^{3} \mathrm{~mol}^{-1}$.

The suggested (by magnetic susceptibility analysis) contribution of DMI was subsequently confirmed by EPR spectroscopy. Indeed, powder X- and Q-band spectra (Figure 2) revealed the presence of an axial signal, consisting of a multiline group at $g \sim 2.25$, assigned to the $g_{\|}$component, and a broad derivative feature at $g \sim 1.5$ assigned to $g_{\perp}$; the position of this latter signal has been analysed by Rakitin on the basis of the relations between $\left|J-J^{\prime}\right|$ and $G_{z}{ }^{26}$ while the width of the signal can be attributed to $J$-strain effects. ${ }^{27}$ These observations are in line with other cases of spin triangles giving rise to isosceles magnetic symmetries $\left(J \neq J^{\prime}\right)$ despite a crystallographically imposed $C_{3 v}$ or $D_{3 h}$ point-group symmetry. ${ }^{28,29}$ 

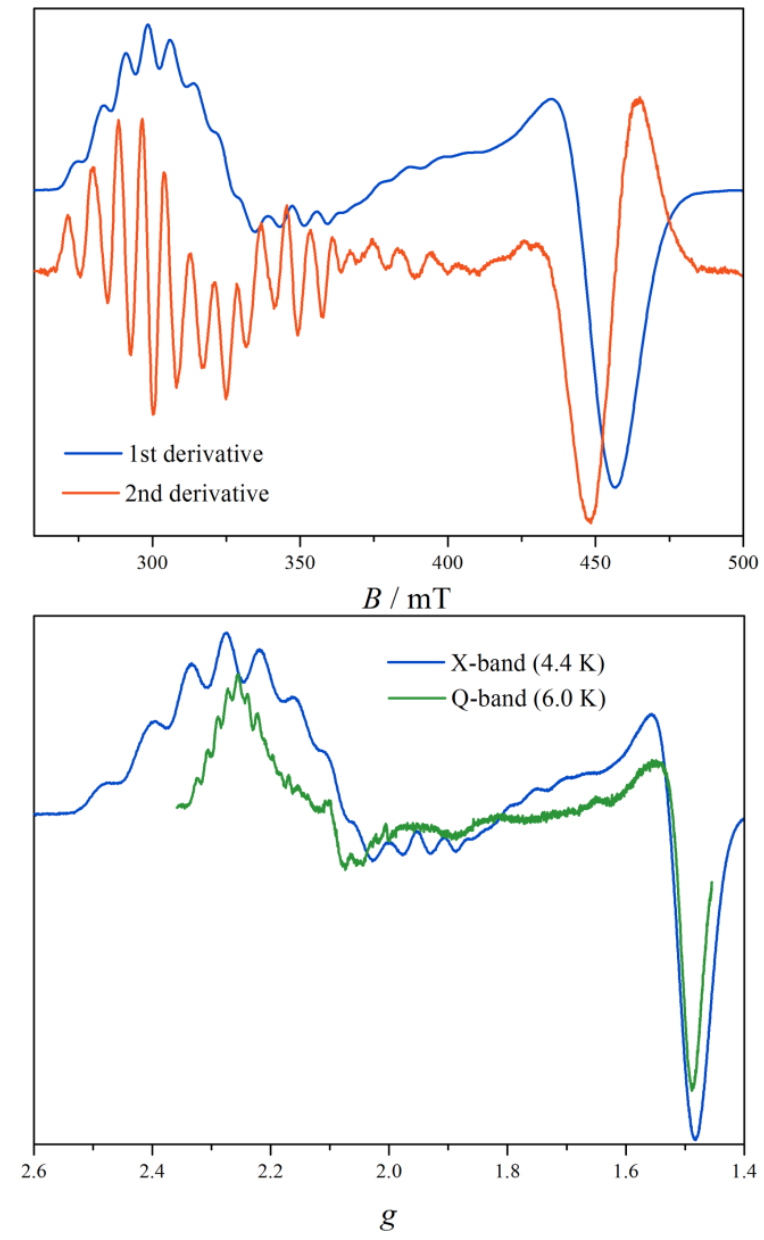

Figure 3. Top: First (blue) and second (orange) derivative X-band powder spectra of 1 at $4.4 \mathrm{~K}$, revealing a multiline pattern. Bottom: Comparison of X-band (blue, $4.4 \mathrm{~K}$ ) and Q-band (green, $6.0 \mathrm{~K}$ ) powder spectra of $\mathbf{1}$ plotted on a common $g$-scale. Exp. Conditions: X-band spectrum, $f_{\mathrm{Mw}}=9.424$ $\mathrm{GHz}, P_{\mathrm{MW}}=2.03 \mathrm{~mW}, B_{\mathrm{mod}}=2 \mathrm{G}_{\mathrm{pp}}$; Q-band spectrum, $f_{\mathrm{MW}}=34.000 \mathrm{GHz}, P_{\mathrm{MW}}=1.17 \mathrm{~mW}, B_{\mathrm{mod}}=5$ $\mathrm{G}_{\mathrm{pp}}$.

To better ascertain the nature and origins of the multiline pattern, experiments were carried out on an indexed single-crystal (Figure S3). Rotations around the $b$-axis, which is parallel to the molecular zaxis, were carried out at both frequencies (Figure 3), and additional rotations around the $c$-axis were carried out at X-band (Figure S4). These latter exhibited neither shifts nor intensity variation upon rotation, confirming, respectively, the coincidence of the molecular z-axis with the magnetic axis and the purely axial character of the signal, with no rhombic contributions.

At X-band $(4.3 \mathrm{~K})$, the $g_{\|}$component consists of a 12-line signal spanning an $88 \mathrm{mT}$ region (264 to $352 \mathrm{mT}$ ), while at Q-band (10 K) a 16-line pattern is clearly defined, spanning a $121 \mathrm{mT}$ (1038 to 1159 $\mathrm{mT}$ ) region (Figure S5). Since the separations of hyperfine features do not change in magnetic field 
terms under different frequencies, this observation can only be interpreted by assuming two slightly different $g_{\|}$resonances, one for each molecule, which are better resolved upon frequency increase.

Rotations of the crystal at the X-band were partially in line with powder spectra, revealing a broad and symmetric resonance at $g=1.5(450 \mathrm{mT})$ for a $90^{\circ}$ rotation $\left(g_{\perp}\right)$. However, they also revealed the existence of another set of resonances of drastically different anisotropy. In particular, at X-band this latter component produced a resonance at $420 \mathrm{mT}$ for a $45^{\circ}$ rotation, disappearing from the observable magnetic fields upon larger rotations. Similar observations were made at the Q-band, where this latter resonance disappeared from the observable magnetic field range for rotations beyond $15^{\circ}$. These two different sets of resonances, of different $g_{\|}$values and different anisotropies, were attributed to the two cocrystallized complex anions of $\mathbf{1 a}$ and $\mathbf{1 b}$.

To quantify the spin Hamiltonian parameters of the two components, we undertook simultaneous fits for various rotations, for both frequencies. While the spectra can be exactly calculated by including a hyperfine term in the aforementioned Hamiltonian, this can be computationally very intensive for EPR spectra calculations; the Hilbert space dimension being $(2 S+1)^{3} \times(2 I+1)^{3}$, for $S=1 / 2$ and $I=3 / 2$ the size of the matrix size is $512 \times 512$. Moreover, this Hamiltonian presupposes the knowledge of the $J$, $J^{\prime}$ and $G_{z}$ parameters, which are indeterminate by bulk methods (e.g. magnetometry), due to the simultaneous presence of both molecules in the sample. It was considered more realistic, both in terms of number of free parameters and of computational cost (128×128 matrix dimensions), to fit the spectra to a mixture of two effective $S=1 / 2\left(S_{\mathrm{A}}\right.$ and $\left.S_{\mathrm{B}}\right)$ systems, each experiencing hyperfine interactions with three $I=3 / 2$ nuclei.

For the calculation of the hyperfine interactions in such a case, we consider that the effective hyperfine tensors are described by $\mathbf{A}_{i}=K_{i} \mathbf{a}_{i}$, where, $\mathbf{a}_{i}$ is the intrinsic hyperfine tensor of each individual ion and $K_{i}$ are coefficients determined by the spin projection on each individual ion. Assuming, for simplicity, only an isosceles distortion and $\left(G_{x}, G_{y}\right) \sim 0$, these coefficients have been analytically calculated as: ${ }^{16}$

$$
\begin{aligned}
& \text { I: } K_{1}=\frac{1}{3}-\frac{2 \delta}{3 \Delta}, K_{2}=K_{3}=\frac{1}{3}\left(1+\frac{\delta}{\Delta}\right) \text { for }|J|>\left|J^{\prime}\right| \\
& \text { II: } K_{1}=\frac{1}{3}+\frac{2 \delta}{3 \Delta}, K_{2}=K_{3}=\frac{1}{3}\left(1-\frac{\delta}{\Delta}\right) \text { for }|J|<\left|J^{\prime}\right|
\end{aligned}
$$

with $\delta / \Delta=1$ corresponding to the isotropic case $(\mathbf{G}=0)$. 
Given the polyparametric nature of the problem, we carried out simulations to obtain a preliminary assessment of the position of the best-fit solution in the parameter space, particularly regarding the $\delta / \Delta$ values. Extensive simulations in the two cases, I and II, for varying $\delta / \Delta$ values ranging from 1 (isotropic system, no DMI) to $\sim 0$ (anisotropic system, strong DMI) are presented as Supporting Information, using the full matrix (Figure S6) or the analytically derived model (Figure S7); their agreement corroborates the analytically derived solutions. An indicative series of simulations using full matrix calculations are shown in Figure 5.

These simulations reveal that for strong DMI both cases converge to a 10-line pattern, assuming a $1.5 \mathrm{mT}$ intrinsic Lorentzian line width. In-between, the progressive regroupings of the 24 resonances into different sets, lead to an intermediate 7-line pattern in case $\mathbf{I}$ (for $\delta / \Delta=0.6-0.5$ ), and to 19-line ( $\delta / \Delta$ $\sim 0.5)$ and 13-line patterns $(\delta / \Delta=0.3-0.2)$ in case $\mathbf{I I}$.

Spectra resulting from these simulations are heavily dependent on the intrinsic line width and the values of the intrinsic hyperfine tensor $\mathbf{a}_{i}$. For comparison, the previously reported 8-line spectra ${ }^{23} \mathrm{can}$ be reproduced by relatively broader $(5 \mathrm{mT})$ lines, with $A_{\|}$values corresponding to $\delta / \Delta=0.26\left(a_{\|} \sim 700\right.$ $\mathrm{MHz})$ or $0.86\left(a_{\|} \sim 620 \mathrm{MHz}\right)$.

Based on the magnetic susceptibility data and the large anisotropies, we considered that relatively strong DMI is at play, making the parameter space of $\delta / \Delta<0.3$ for both components more physically meaningful. Successive simulations gave consistently better results for a $|J|>\left|J^{\prime}\right|$ (case I) magnetic symmetry. Fits based on this starting point, gave a satisfactory solution with parameters: $g_{\| \mathrm{A}}=2.206$, $g_{\perp \mathrm{A}}=1.48, \delta_{\mathrm{A}} / \Delta_{\mathrm{A}}=0.251, \sigma g_{\perp \mathrm{A}}=0.03, A_{\| \mathrm{A}}=610.3 \mathrm{MHz}$ for (low anisotropy) component $\mathbf{A}$ and $g_{\| \mathrm{B}}=$ 2.113, $g_{\perp \mathrm{B}}=0.267, \delta_{\mathrm{B}} / \Delta_{\mathrm{B}}=0.077, \sigma g_{\perp \mathrm{B}}=0.008, A_{\| \mathrm{B}}=588.8 \mathrm{MHz}$ for (high anisotropy) component $\mathrm{B}$.

We have previously explained the broader lines at $90^{\circ}$ rotations by the effect of $J$-strain effects under the influence of DMI on the $g_{\perp}$ values, and we have demonstrated empirical ${ }^{27}$ and explicit ${ }^{29}$ methods of taking those into consideration. However, given the size of the problem at hand, and the computational overhead these methods introduce due to the need to calculate large numbers of variations of the system, we were unable to employ them here. Instead, the $g_{\perp}$ line broadenings were modeled using Easyspin's $g$-strain modeling, which explains the imperfect reproduction of the features at $90^{\circ}$ rotations. 


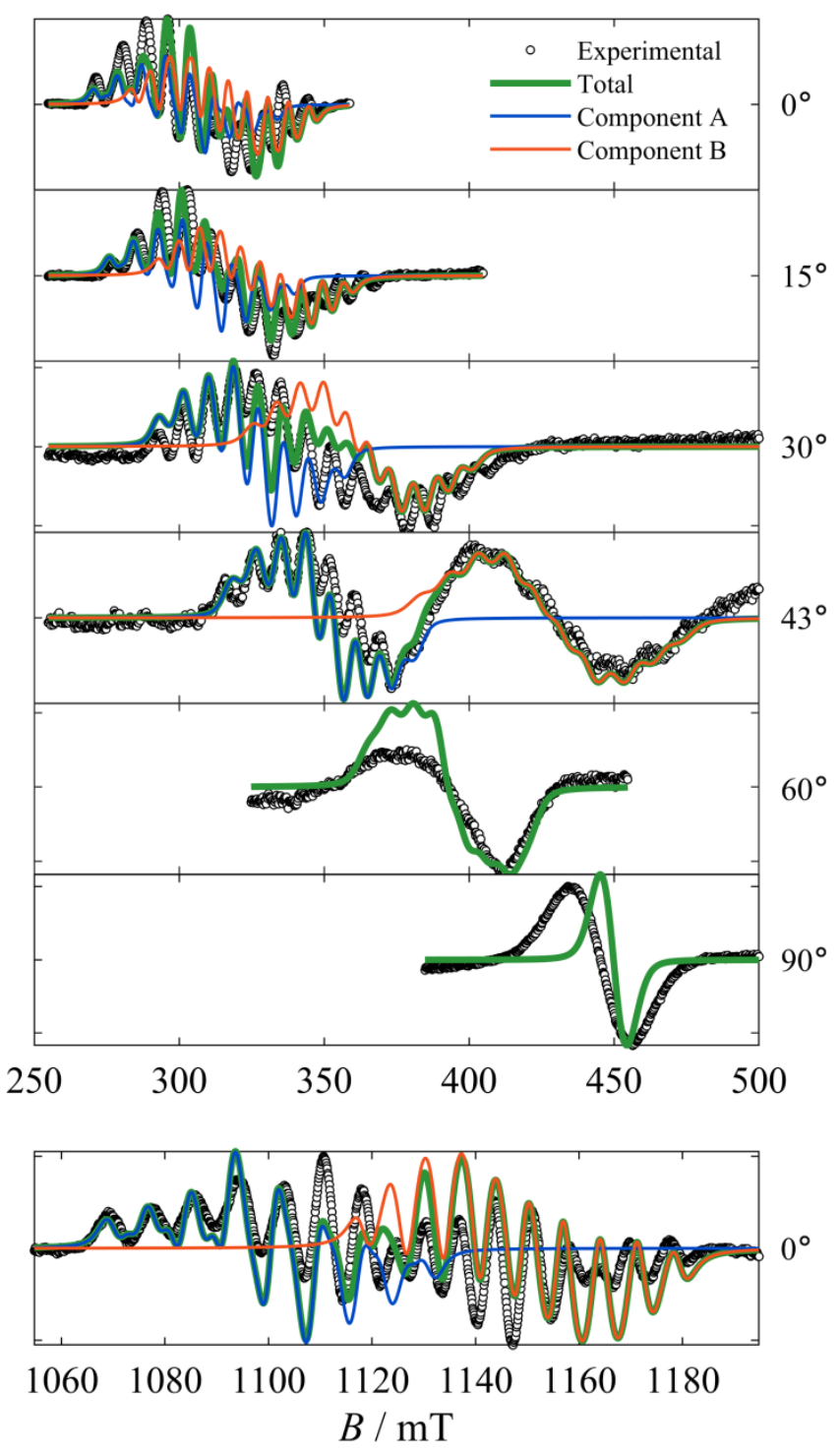

Figure 4. Simultaneous fits to multirotational dual-frequency single-crystal data of $\mathbf{1}$, with best-fit parameters as mentioned in the text. Top panel: X-band data. Exp. Conditions: $T=4.3 \mathrm{~K}, f_{\mathrm{MW}}=9.328$ $\mathrm{GHz}, P_{\mathrm{MW}}=4.58 \mathrm{~mW}, B_{\mathrm{mod}}=10 \mathrm{G}_{\mathrm{pp}}$. Bottom panel: Q-band data at $0^{\circ} . T=10 \mathrm{~K}, f_{\mathrm{MW}}=33.978 \mathrm{GHz}$, $P_{\mathrm{MW}}=2.51 \mathrm{~mW}, B_{\mathrm{mod}}=10 \mathrm{G}_{\mathrm{pp}}$.

To assess these results some comments are in order regarding the model used: It does render the calculation computationally feasible, but at the cost of certain simplifications. In particular, equations (2) and (3) assume that the single-ion g-tensor z-components are all parallel to the molecular z-axis. However, tilts of these tensors with respect to the molecular $z$-axis by an angle $\alpha$ would modify the local d-functions and their concomitant effects on the electron transfer integrals induce an in-plane DMI component, $G_{X} \neq 0 .^{30}$ In addition, when such tilts occur, the effective $g$ values of the exchange- 
coupled system are no longer described by relations (2) and (3). Simulations show that $g_{\|, \text {eff }}$ varies as $g_{\|, \text {eff }}^{2}=g_{0 \|}{ }^{2} \cos ^{2}(\alpha)+g_{0 \perp}{ }^{2} \sin ^{2}(\alpha)$, i.e. as the tilt angle brings the $z$-axes of the local tensors closer to the triangle plane, it is the $g_{0 \perp}$ component that is predominantly projected on the value of $g_{\| \text {,eff, as we }}$ previously observed in a family of ferromagnetic copper(II) pyrazolato triangles. ${ }^{17}$

Equations (3) and (4), in turn, assume that g- and A-tensors are collinear with their z-axes parallel to the molecular z-axis. However, tilts of the local axes such as mentioned above produce a similar effect on the affective hyperfine coupling: assuming $A_{\|}>A_{\perp} \sim 0$, as is commonly the case for $\mathrm{Cu}^{\mathrm{II}}$ ions, increase of angle $\alpha$ leads to a gradual narrowing of the effective hyperfine patterns at $\mathbf{B}_{0} \| z$, until their complete collapse at $\alpha=90^{\circ}$.

These remarks can be used to rationalize the observation of two slightly different $g_{\| \text {,eff }}$ values, initially revealed by dual-frequency spectra at zero rotation (see above): as the out-of-plane positions of the $\mu_{3}-\mathrm{Cl}^{-}$and $\mu_{3}-\mathrm{OH}^{-}$ligands in $\mathbf{1 b}$ tilt the local g-tensors, the effective value $g_{\| \mathrm{B}}$ is slightly reduced, since for copper(II) ions in octahedral or square-planar environments $g_{0 \|}>g_{0 \perp}$. Indeed, assuming that $g_{0 \|}=2.22$ and $g_{0 \perp}=2.05$, an angle $\alpha \sim 50^{\circ}$ would yield $g_{\|, \text {eff }} \sim 2.12$, very close to the one derived for $g_{\| \mathrm{B}}$ from the fits (vide supra). This value is close to the $\mathrm{O}(1 \mathrm{~A})-\mathrm{Cl}(0 \mathrm{~A})-\mathrm{Cu}(1)$ angle of $43.4^{\circ}$, indicating that this is a satisfactory qualitative measure of the local tensor tilt angle, although theoretical calculations would be needed for a more precise determination of its orientation. Based on this reasoning, component $\mathbf{B}$, characterized by a lower $g_{\|}$value, is assigned to molecule $\mathbf{1} \mathbf{b}$, an assignment also in agreement with its lower $A_{\|}$value. This assignment also allows us to associate the higher anisotropy component $\mathbf{B}$ to molecule $\mathbf{1 b}$. 


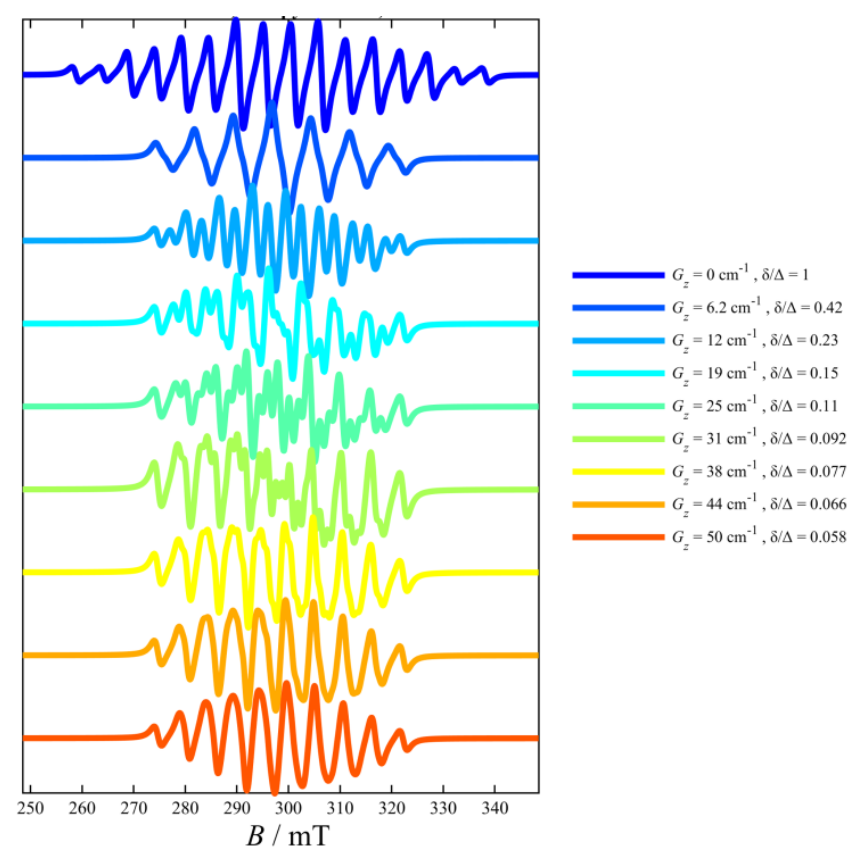

Figure 5. Simulations of the evolution of the multiline hyperfine pattern with increasing DMI (from top to bottom) based on full-matrix calculations of the spectra for $J=-50 \mathrm{~cm}^{-1}$ and $\Delta J=-5 \mathrm{~cm}^{-1}(<0$, case $\mathbf{I})$, which leads to a 16-line pattern in the isotropic case. Narrower variations of $G_{z}$ for both cases (I and II) are shown in Figures S6 (full-matrix calculations) and S7 (calculations based on analytical expressions).

From a quantitative perspective, the fits exhibit disagreements with the experimental data, which are unavoidable given the simplifications we introduced to our model; more detailed conclusions would require full-matrix calculations. These are less straightforward due to the coexistence of the two species, which impedes us from estimating their $J$ and $G_{z}$ values. Additional complications arise from the size of the full Hamiltonian matrix, which renders the problem computationally intensive, as well as the difficulty in determining the precise orientations of the $g$ - and A-tensors, which would require the use of advanced $a b$ initio calculations. Finally, our model considers the $\mathbf{G}$ pseudovector parallel to the molecular z-axis $\left(G_{x}=G_{y}=0\right)$. However, as the local $g$-tensors of the metal ions are tilted with respect to the molecular z-axis, Moriya's rules ${ }^{31}$ applied to the pairs of $\mathrm{Cu}$ atoms, reveal only a mirror plane dissecting the intermetallic vector. Thus, $\mathbf{G}$ will lie in that plane as per Moriya's second rule, i.e. it will be characterized by a $z$ - and by an $x$-component.

In conclusion, in this work we analyzed the influence of DMI on the hyperfine coupling pattern of a molecular spin triangle, the first such demonstration in a molecular material, both theoretically and experimentally. Moreover, we achieved this demonstration for a rare case of a double salt, containing 
two magnetically distinct complex anions, by analyzing the hyperfine patterns of single-crystal dualfrequency EPR spectra.

\section{Experimental}

Full details of the materials, methods, and crystal structure parameters for complex $\mathbf{1}$ can be found in the Supporting Information. Deposition Number 2067279 contains the supplementary crystallographic data for this paper. These data are provided free of charge by the joint Cambridge Crystallographic Data Centre and Fachinformationszentrum Karlsruhe Access Structures service www.ccdc.cam.ac.uk/structures.

\section{Acknowledgements}

This project has received funding from the European Union's Horizon 2020 research and innovation programme under the Marie Sklodowska-Curie grant agreement No 746060 (project “CHIRALQUBIT”). We thank Dr. Lydia Karmazin and Dr. Corinne Bailly for help with indexing of the single crystal.

\section{References}

1 M. Trif, F. Troiani, D. Stepanenko and D. Loss, Spin-Electric Coupling in Molecular Magnets, Phys. Rev. Lett., 2008, 101, 217201.

2 M. Trif, F. Troiani, D. Stepanenko and D. Loss, Spin electric effects in molecular antiferromagnets, Phys. Rev. B, 2010, 82, 045429.

3 F. Troiani, D. Stepanenko and D. Loss, Hyperfine-induced decoherence in triangular spin-cluster qubits, Phys. Rev. B, 2012, 86, 161409(R).

4 A. K. Boudalis, J. Robert and P. Turek, First Demonstration of Magnetoelectric Coupling in a Polynuclear Molecular Nanomagnet: Single-Crystal EPR Studies of $\left[\mathrm{Fe}_{3} \mathrm{O}\left(\mathrm{O}_{2} \mathrm{CPh}\right)_{6}(\mathrm{py})_{3}\right] \mathrm{ClO}_{4} \cdot \mathrm{py}$ under Static Electric Fields, Chem. - Eur. J., 2018, 24, 14896-14900.

5 J. Liu, J. Mrozek, W. K. Myers, G. A. Timco, R. E. P. Winpenny, B. Kintzel, W. Plass and A. Ardavan, Electric Field Control of Spins in Molecular Magnets, Phys. Rev. Lett., 2019, 122, 037202.

6 J. Robert, N. Parizel, P. Turek and A. K. Boudalis, Polyanisotropic Magnetoelectric Coupling in an Electrically Controlled Molecular Spin Qubit, J. Am. Chem. Soc., 2019, 141, 19765-19775.

7 B. Kintzel, M. Fittipaldi, M. Böhme, A. Cini, L. Tesi, A. Buchholz, R. Sessoli and W. Plass, SpinElectric Coupling in a Cobalt(II)-Based Spin Triangle Revealed by Electric Field-Modulated ESR, Angew. Chem., , DOI:10.1002/ange.202017116.

8 M. I. Belinsky, Field-dependent spin chirality and frustration in $\mathrm{V}_{3}$ and $\mathrm{Cu}_{3}$ nanomagnets in transverse magnetic field. 1 . Correlations between variable planar spin configurations, vector and scalar chiralities and magnetization, Chem. Phys., 2014, 435, 62-94. 
9 M. I. Belinsky, Field-dependent spin chirality and frustration in nanomagnets in transverse magnetic field. 2. Spin configurations, chirality and intermediate spin magnetization in distorted trimers, Chem. Phys., 2014, 435, 95-125.

10K. Kambe, On the Paramagnetic Susceptibilities of Some Polynuclear Complex Salts, J. Phys. Soc. Jpn., 1950, 5, 48-51.

11J. Wucher and J. D. Wasscher, Couplage De Spins et Anomalies de Chaleur Spécifique aux Températures de l'Hélium et de l'Hydrogène Liquides Pour l'Acétate Complexe $\left[\mathrm{Cr}_{3}\left(\mathrm{CH}_{3} \mathrm{COO}\right)_{6}(\mathrm{OH})_{2}\right] \mathrm{Cl} .8 \mathrm{H}_{2} \mathrm{O}$, Physica, 1954, 20, 721-726.

12 Yu. V. Yablokov, V. A. Gaponenko, V. V. Zelentsov and K. M. Suvorova, Paramagnetic resonance of some three-nuclear clusters of iron, Solid State Commun., 1974, 14, 131-135.

13A. Bencini, D. Gatteschi and A. Bencini, EPR of exchange coupled systems, Dover Publications, Inc, Mineola, N.Y, Dover ed., 2012.

14 M. I. Belinsky, Hyperfine Splittings in Spin-Frustrated Trinuclear Cu 3 Clusters, Inorg. Chem., 2004, 43, 739-746.

15A. K. Boudalis, Y. Sanakis, C. P. Raptopoulou, A. Terzis, J.-P. Tuchagues and S. P. Perlepes, A trinuclear cluster containing the $\left\{\mathrm{Fe}_{3}\left(\mu_{3}-\mathrm{O}\right)\right\}^{7+}$ core: Structural, magnetic and spectroscopic (IR, Mössbauer, EPR) studies, Polyhedron, 2005, 24, 1540-1548.

16L. Mathivathanan, A. K. Boudalis, P. Turek, M. Pissas, Y. Sanakis and R. G. Raptis, Interactions between $\mathrm{H}$-bonded $\left[\mathrm{Cu}_{3}^{\mathrm{II}}\left(\mu_{3}-\mathrm{OH}\right)\right]$ triangles; a combined magnetic susceptibility and EPR study, Phys. Chem. Chem. Phys., 2018, 20, 17234-17244.

17L. Mathivathanan, G. Rogez, N. Ben Amor, V. Robert, R. G. Raptis and A. K. Boudalis, Origin of Ferromagnetism and Magnetic Anisotropy in a Family of Copper(II) Triangles, Chem. - Eur. J., 2020, 26, 12769-12784.

18A. Boudalis, Half-integer spin triangles: old dogs, new tricks, Chem. - Eur. J., , DOI:10.1002/chem.202004919.

19B. Tsukerblat, M. Belinskii and V. Fainzil'berg, Magnetochemistry and spectroscopy of transition metal exchange clusters, Sov. Sci Rev B Harwood Acad Pub, 1987, 337-482.

20 J. Yoon, L. M. Mirica, T. D. P. Stack and E. I. Solomon, Spectroscopic Demonstration of a Large Antisymmetric Exchange Contribution to the Spin-Frustrated Ground State of a $D_{3}$ Symmetric Hydroxy-Bridged Trinuclear Cu(II) Complex: Ground-to-Excited State Superexchange Pathways, $J$. Am. Chem. Soc., 2004, 126, 12586-12595.

21B. Cage, F. A. Cotton, N. S. Dalal, E. A. Hillard, B. Rakvin and C. M. Ramsey, Observation of Symmetry Lowering and Electron Localization in the Doublet-States of a Spin-Frustrated Equilateral Triangular Lattice: $\mathrm{Cu}_{3}\left(\mathrm{O}_{2} \mathrm{C}_{16} \mathrm{H}_{23}\right) \cdot 1.2 \mathrm{C}_{6} \mathrm{H}_{12}$, J. Am. Chem. Soc., 2003, 125, 5270-5271.

22 G. Mezei, R. G. Raptis and J. Telser, Trinuclear, Antiferromagnetically Coupled $\mathrm{Cu}^{\mathrm{II}} \mathrm{Complex}$ with an EPR Spectrum of Mononuclear Cu ${ }^{\text {II }}$ : Effect of Alcoholic Solvents, Inorg. Chem., 2006, 45, 8841-8843.

23X. Liu, J. A. McAllister, M. P. de Miranda, E. J. L. McInnes, C. A. Kilner and M. A. Halcrow, Reactions of Copper(II) Salts with 3\{5\}-tert-Butylpyrazole: Double-Cubane Complexes with Bound Exogenous Anions, and a Novel Pyrazole Coordination Mode, Chem. - Eur. J., 2004, 10, 18271837.

24 Y. Sanakis, A. L. Macedo, I. Moura, J. J. G. Moura, V. Papaefthymiou and E. Münck, Evidence for Antisymmetric Exchange in Cuboidal [3Fe-4S ${ }^{+}$Clusters, J. Am. Chem. Soc., 2000, 122, 1185511863. 
25M. Rivera-Carrillo, I. Chakraborty, G. Mezei, R. D. Webster and R. G. Raptis, Tuning of the [Cu $3(\mu-$ O) $]^{4+/ 5^{+}}$Redox Couple: Spectroscopic Evidence of Charge Delocalization in the Mixed-Valent $\left[\mathrm{Cu}_{3}(\mu-\mathrm{O})\right]^{5+}$ Species, Inorg. Chem., 2008, 47, 7644-7650.

26 Yu. V. Rakitin, Yu. V. Yablokov and V. V. Zelentsov, EPR spectra of trigonal clusters, J. Magn. Reson. 1969, 1981, 43, 288-301.

27Y. Sanakis, A. K. Boudalis and J.-P. Tuchagues, J-strain and antisymmetric exchange in a polynuclear compound containing the $\left\{\mathrm{Fe}_{3} \mathrm{O}\right\}^{7+}$ core, Comptes Rendus Chim., 2007, 10, 116-124.

28 A. N. Georgopoulou, I. Margiolaki, V. Psycharis and A. K. Boudalis, Dynamic versus Static Character of the Magnetic Jahn-Teller Effect: Magnetostructural Studies of $\left[\mathrm{Fe}_{3} \mathrm{O}\left(\mathrm{O}_{2} \mathrm{CPh}\right)_{6}(\mathrm{py})_{3}\right] \mathrm{ClO}_{4} \cdot \mathrm{py}$, Inorg. Chem., 2017, 56, 762-772.

29A. K. Boudalis, G. Rogez and P. Turek, Determination of the Distributions of the Spin-Hamiltonian Parameters in Spin Triangles: A Combined Magnetic Susceptometry and Electron Paramagnetic Resonance Spectroscopic Study of the Highly Symmetric $\left[\mathrm{Cr}_{3} \mathrm{O}(\mathrm{PhCOO})_{6}(\mathrm{py})_{3}\right]\left(\mathrm{ClO}_{4}\right) \cdot 0.5 \mathrm{py}$, Inorg. Chem., 2018, 57, 13259-13269.

30 M. I. Belinsky, Spin-Frustrated Trinuclear Cu(II) Clusters with Mixing of 2(S = 1/2) and S = 3/2 States by Antisymmetric Exchange. 2. Orbital Origin of In-Plane Dzialoshinsky-Moriya Exchange Parameters, Inorg. Chem., 2008, 47, 3532-3539.

31 T. Moriya, Anisotropic Superexchange Interaction and Weak Ferromagnetism, Phys. Rev., 1960, 120, 91-98. 


\section{Table of contents entry}

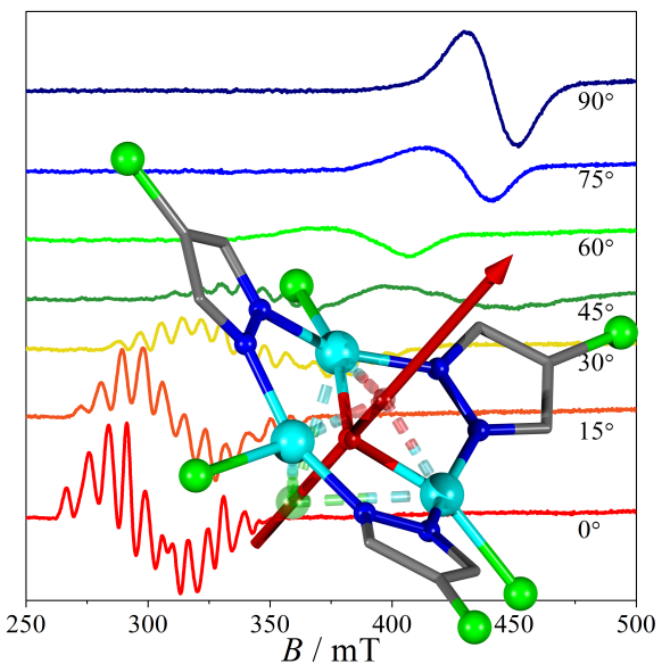

\section{Keywords}

Cluster compounds; EPR spectroscopy; Dzyaloshinskii-Moriya interactions; Hyperfine interactions; Spin triangles 